\title{
PRESCRIPTION PATTERN IN OBESE AND NON-OBESE INFERTILE WOMEN WITH POLYCYSTIC OVARY SYNDROME IN A TERTIARY CARE HOSPITAL
}

\author{
ASHA AVIRAH MM ${ }^{1 *}$, ASWATHY ALIAS ${ }^{1}$, MANJUSHA SAJITH ${ }^{1}$, VANDANA NIMBARGI ${ }^{2}$, SHIVHAR KUMDALE ${ }^{1}$ \\ ${ }^{1}$ Department of Clinical Pharmacy, Bharati Vidyapeeth Deemed University, Poona College of Pharmacy, Pune, Maharashtra, \\ India. ${ }^{2}$ Department of Obstetrics and Gynaecology, Bharati Hospital and Research Centre, Pune, Maharashtra, India
}

Email: Manjusaji1@yahoo.com

Received: 10 October 2017, Revised and Accepted: 23 November 2017

ABSTRACT

Objective: The objective of this study is to evaluate the treatment options for the management of obese and non-obese infertile women with polycystic ovary syndrome (PCOS).

Methods: A prospective observational study was conducted with 75 infertile PCOS women. The demographic details, body mass index, menstrual patterns, and current medication related to infertility were noted. The collected data were statistically represented in terms of range, frequency tables, and standard deviation wherever appropriate.

Results: A majority of the infertile PCOS women were aged 24-27 years with a mean age of $25.72 \pm 3.53$ years, belonging to middle socioeconomic class (44\%) and mostly found to be urban residents 68\%. Most of the PCOS women were overweight (32\%) and obese (21\%) with irregular menstrual pattern (90.67\%). In combination therapy, clomiphene citrate (CC) pre-treated with oral contraceptives (OC) (37.93\%) was mostly given to obese PCOS patients followed by CC with metformin pre-administered with OC (31.03\%), whilen on-obese PCOS patients were mostly administered CC with gonadotropins pre-treated with OC(44\%). In single therapy, OC was mostly administered to both non-obese (90\%) and obese PCOS patients $(88.90 \%)$.

Conclusion: This study concluded that most of the infertile PCOS women were overweight and obese, a major risk actor causing hyperandrogenicity. CC pre-treated with OC and metformin with CC pre-treated with OC were mostly prescribed to obese PCOS patients. Lifestyle modifications along with treatment are strongly recommended, especially in obese PCOS patients.

Keywords: Polycystic ovary syndrome, Overweight, Obese, Oral contraceptives, Clomiphene citrate, Metformin.

(c) 2018 The Authors. Published by Innovare Academic Sciences Pvt Ltd. This is an open access article under the CC BY license (http://creativecommons. org/licenses/by/4. 0/) DOI: http://dx.doi.org/10.22159/ajpcr.2018.v11i3.22936

\section{INTRODUCTION}

Polycystic ovary syndrome (PCOS), one of the leading causes of infertility known today, is a as oligomenorrhea, amenorrhea, hirsutism, acne, alopecia, and obesity [1].

PCOS has a prevalence of $5-10 \%$ in women of childbearing age with variance among races, ethnicities, and geographical areas [2,3]. The highest reported a prevalence of $52 \%$ has been among the South Asian immigrants in Britain, of which $30-75 \%$ were obese [4]. Obesity is known to worsen the clinical, endocrine, and metabolic features of the syndrome by increasing insulin resistance and hyperinsulinemia and further causing hyperandrogenicity [5-7].

Management of PCOS in infertile women is mostly individualized and symptom oriented. The main objectives are to reinstate normal menstrual and ovulatory cycle along with fertility in terms of conception, to treat acne, hirsutism, and infertility, and to prevent weight gain and endometrial carcinoma. Currently, the commonly used regimens are clomiphene citrate (CC), metformin, oral contraceptives (OC), gonadotropins, and laparoscopic ovarian drilling (LOD); a minimally invasive surgery [8].

In today's era, infertility due to PCOS is becoming more common mainly because of their unhealthy lifestyle habits such as sedentary habits and junk food and many other unknown reasons. This study aimed at determining the treatment options in the management of PCOS in obese and non-obese women with infertility. Therefore, it is hoped that the findings from this study will enable us to determine the different therapeutic options for PCOS in our locality since there is a paucity of knowledge in the provision of such care in our environment.

\section{METHODS}

A prospective case-control observational study was conducted in Bharati Hospital and Research Center Pune, Pune, over 9 months from August 2016 to April 2017 which included 75 infertile PCOS women.

The study was approved by the Institutional Ethics Committee, and before initiating the study, informed consent was taken from all patients participating in the study. Women of reproductive age (20-39 years) who are attending or admitted with complaints and symptoms suggesting PCOS leading to infertility were included in the study.

The details such as age, employment status, socioeconomic status, area of residence, body mass index (BMI), menstrual history, past medical history, past medication history, and current medication (number of medications, dose regimen, and frequency) related to infertility were noted from the fertility outpatient department (OPD) case paper sheets in the self-pre-designed patient profile form. Socioeconomic class and employment status were determined based on patient's education, husband's occupation, and monthly income. The BMI was calculated based on the height and weight revealed in the fertility OPD case sheets.

The collected data were analyzed for the various treatment options in obese and non-obese PCOS infertile women. The data were statistically represented in terms of range, mean, frequency tables, and standard deviation wherever appropriate. 


\section{RESULTS}

Sociodemographic characteristics in infertile women with PCOS Of 75 PCOS infertile women studied, most of the women were aged 24-27 years with a mean age of $25.72 \pm 3.53$ years. Majority of the PCOS women were found to be housewives (61.33\%), from a middle socioeconomic class (44\%), and mostly residing in urban areas (68\%) (Table 1).

Menstrual pattern, duration of infertility, and types of infertility in infertile women with PCOS

Table 2 presents that most of the infertile PCOS women were having irregular menstrual pattern $(90.67 \%)$ with an average duration of infertility $\leq 2$ years $(77.33 \%)$. Primary infertility was most common type of infertility seen in PCOS women (22.67\%).

\section{BMI of infertile women with PCOS}

Fig. 1 describes that most of the infertile PCOS women had obesity (53\%) wherein $32 \%$ were overweight and $21 \%$ obese. $35 \%$ of the women had normal weight.

Frequencies of treatments used in the management of non-obese and obese infertile women with PCOS

Majority of the non-obese PCOS women were administered with OC (97\%), followed by CC (65.71\%) and gonadotropins $(42.86 \%)$, whereas most of the obese PCOS women were given diet and exercise (100\%), followed by OC (90.00\%), CC (72.50\%), metformin (27.50\%), and LOD (10\%) (Fig. 2).

\section{Prescription pattern in non-obese and obese infertile women with} PCOS

Table 3 describes that, of 75 PCOS patients in this study, 54 patients were given combination therapy $(72.00 \%)$ while only 19 patients were

Table 1: Sociodemographic characteristics in infertile women with PCOS

\begin{tabular}{ll}
\hline Characteristics & Number of PCOS patients $(\mathbf{n = 7 5})$ \\
\hline Age (years) & $20(26.67)$ \\
$20-23$ & $33(44.00)$ \\
$24-27$ & $15(20.00)$ \\
$28-31$ & $7(9.33)$ \\
$32-35$ & 0 \\
$36-39$ & $33(44.00)$ \\
Socioeconomic class & $25(33.33)$ \\
Middle & $17(22.67)$ \\
Low & $46(61.33)$ \\
High & $29(38.67)$ \\
Employment status & \\
House wife & \\
Employed & \\
Area of residence & \\
Urban & $24(68.00)$ \\
Rural & \\
\hline
\end{tabular}

PCOS: Polycystic ovary syndrome

Table 2: Menstrual pattern, duration of infertility, and types of infertility in non-obese and obese women with PCOS

\begin{tabular}{ll}
\hline Parameters & Number of PCOS patients (n=75) \\
\hline Menstrual cycle & \\
$\quad$ Irregular & $68(90.67)$ \\
$\quad$ Regular & $7(9.33)$ \\
Duration of Infertility & \\
$\quad \leq 2$ years & $58(77.33)$ \\
$\quad$ More than 2 years & $17(22.67)$ \\
Types of infertility & \\
$\quad$ Primary & $51(68.00)$ \\
$\quad$ Secondary & $24(32 \mathrm{~s} .00)$ \\
\hline PCOS: Polycystic ovary syndrome &
\end{tabular}

given single therapy $(25.33 \%)$. In combination therapy, CC pre-treated with OC (37.93\%) was mostly given to obese PCOS infertile patients followed by CC with metformin pre-administered with OC (31.03\%). Non-obese PCOS infertile patients were mostly administered CC with gonadotropins pre-treated with OC (44\%). In a single therapy, OC was mostly administered to both non-obese $(90 \%)$ and obese PCOS infertile patients (88.90\%).

\section{DISCUSSION}

In this study, most of the infertile PCOS women were aged 24-27 years $(29.33 \%)$ with a mean age of $25.72 \pm 3.53$ years. However, in India, this is not surprising as most of the women get married in their early or midtwenties and hence shows an early need for conception. The result of the PCOS study group is similar when compared to Vrunda et al. [8] who reported that most of the infertile PCOS women were aged 21-26 years $(58 \%)$ with a mean age of 25 years.

Many studies have revealed that lower and middle socioeconomic classes have increased PCOS rates due to stress related to financial hardships and treatment, poor nutritional diet, poor quality of life, and impaired health due to unhealthy lifestyle habits [8-10]. In this study, majority of the infertile PCOS women were from a middle socioeconomic class (44\%). This study result is higher as compared to that reported by Dasgupta and Reddy, in Hyderabad, India [11], wherein most of the PCOS women were from low-middle socioeconomic class (38.4\%). In this study, the prevalence PCOS women (65.71\%) in urban areas were found to be higher than their rural counterparts. The reason behind this may be a sedentary lifestyle and unhealthy dietary habits mainly including junk foods. The study result is consistent with that the study reported by Sarkar et al. [12] in 2006 who revealed that lifestyle changes including lack of exercise and low physical activity play a role in the prevalence of PCOS to be more in urban than rural areas.

Primary infertility (68\%) in infertile PCOS women was found to be higher than secondary infertility $(32 \%)$ in this study. Similarly, a study conducted by Vrunda et al. [8] also reported a higher incidence of primary infertility $(70 \%)$ than secondary infertility. Furthermore,

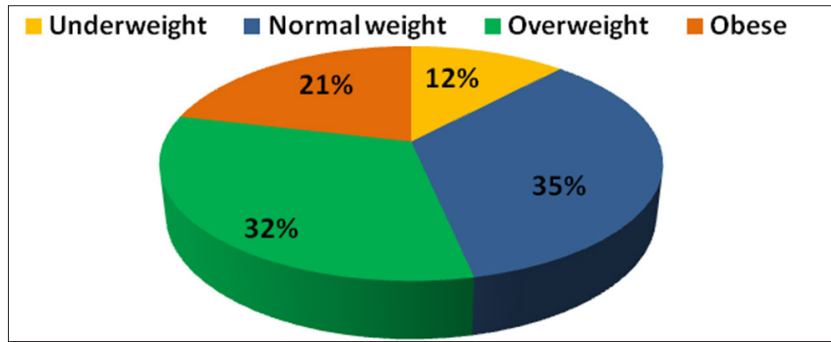

Fig. 1: Body mass index of infertile women with polycystic ovary syndrome

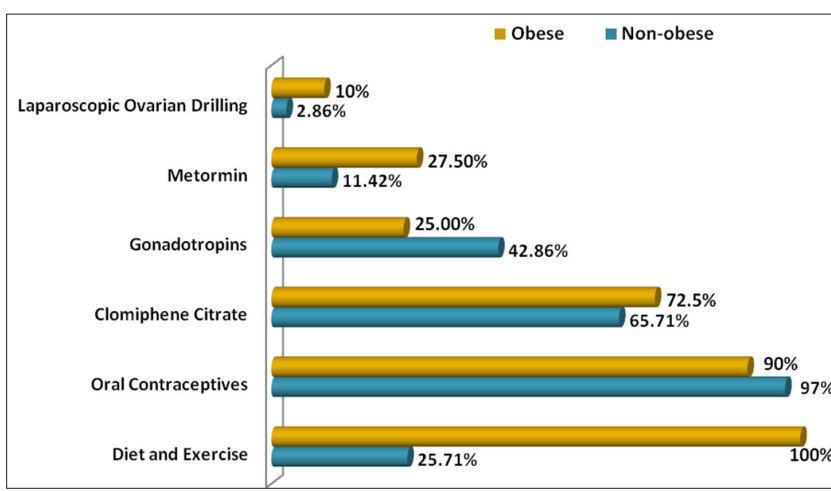

Fig. 2: Frequencies of treatments used in the management of nonobese and obese infertile women with polycystic ovary syndrome 
Table 3: Prescription pattern in non-obese and obese infertile women with PCOS

\begin{tabular}{lll}
\hline Treatment options & Non-obese (n=35) & Obese (n=40) \\
\hline Diet and exercise : & $0(0.00)$ & $2(5.00)$ \\
Single therapy: & $10(28.57)$ & $9(22.5)$ \\
OC & $9(90.00)$ & $8(88.90)$ \\
CC & $0(0.00)$ & $1(11.11)$ \\
Gonadotropins & $1(10.00)$ & $0(0.00)$ \\
Combination therapy: & $25(71.42)$ & $29(72.50)$ \\
OC/CC & $7(28.00)$ & $11(37.93)$ \\
OC/CC/gonadotropin & $11(44.00)$ & $2(6.90)$ \\
OC/CC/gonadotropin/ & $3(12.00)$ & $1(3.45)$ \\
metformin & & \\
OC/CC/metformin & $1(4.00)$ & $9(31.03)$ \\
OC/CC/gonadotropin/ & $1(4.00)$ & $2(6.90)$ \\
LOD & & \\
OC/gonadotropin & $1(4.00)$ & $1(3.45)$ \\
OC/metformin & $0(0.00)$ & $1(3.45)$ \\
CC/gonadotropin & $0(0.00)$ & $1(3.45)$ \\
OC/CC/LOD & $1(4.00)$ & $0(0.00)$ \\
OC/CC gonadotropin/ & $0(0.00)$ & $1(3.45)$
\end{tabular}

metformin/LOD

*(/)-Following. LOD: laparoscopic ovarian drilling, OC: Oral contraceptives,

CC: Clomiphene citrate, PCOS: Polycystic ovary syndrome

the duration of infertility (71.67\%) was mostly found $\leq 2$ years in PCOS women. The apparent underlying reasons for infertility can be multifactorial such as persistent anovulation over a prolonged period, delay in seeking medical help, and expensive treatments.

Obese women are more prone to have irregularity in their menstrual cycles and anovulatory infertility than women with normal BMI. This study reports that $53 \%$ of PCOS patients had high BMI, wherein $32 \%$ were overweight and $21 \%$ were obese. The reason behind this can be the deranged metabolism, lack of exercise, unhealthy dietary habits, especially junk foods, impaired glucose tolerance, and a greater degree of insulin resistance in women with PCOS. This result is in good agreement with the study conducted in New Delhi [13] which showed that $58 \%$ of the patients had high BMI, wherein $38 \%$ were overweight and $20 \%$ obese.

Lifestyle modifications including diet and exercise are recommended as the first-line therapy of PCOS, especially in overweight and obese women as it improves ovulation as well as pregnancy rates and decreases testosterone levels [8,14]. In this study, $100 \%$ of the obese PCOS group was recommended diet and exercise, while only $25.71 \%$ of the non-obese PCOS group was recommended lifestyle modifications.

OCs are hormonal drugs prescribed as the first-line treatment by most infertility consultants as they are effective in regularizing menstrual cycle (downregulation) as well as in the treatment of hirsutism and acne [15]. OCs are also given as a pre-treatment pill for clomipheneresistant patients as it improves ovulation induction as well as pregnancy rates [14]. CC is the recommended first-line treatment for ovulation induction [8]. The use of gonadotropin following CC was postulated by Kistner [16] in 1966 to improve ovulation as well as pregnancy rates as gonadotropin increases the number of pre-ovulatory follicles. Metformin plays a major role in improving ovulation induction in women with PCOS by decreasing the insulin levels or increasing insulin sensitivity and thus altering the effect of insulin on ovarian androgen biosynthesis, theca proliferation, and endometrial growth [17]. Therefore, it increases ovulation rate and pregnancy rate in patients resistant to $\mathrm{CC}$ alone. It is also recommended as first-line therapy in obese patients. Another treatment option for clomiphene-resistant anovulatory PCOS is LOD or diathermy and is a specific treatment option only for PCOS [8,14]. Letrozole, an aromatase inhibitor (for ovulation induction) that does not possess the adverse anti-estrogenic effects of CC, is used in the treatment of infertile PCOS patients [18].
Of 75 PCOS patients in this study, 54 patients were given combination therapy $(72.00 \%)$, while only 19 patients were given single therapy (25.33\%). In single therapy, OC was mostly administered to both nonobese $(90 \%)$ and obese PCOS patients (88.90\%). One of the reasons for the choice of $\mathrm{OC}$ is that it reduces ovarian hyperstimulation (downregulation treatment cycle). CC and gonadotropins alone were also given but less frequently. In combination therapy, CC pre-treated with OC $(37.93 \%)$ was mostly given to obese PCOS patients followed by CC with metformin pre-administered with OC (31.03\%). Non-obese patients were mostly administered CC with gonadotropins pre-treated with OC (44\%). Other combinations such as metformin pre-treated with OC, gonadotropin pre-treated with OC, and CC with gonadotropin pre-administered with OC along with LOD (a minimal invasive surgery) were less frequently used.

Limitations of our study include a small sample size and short duration of the study. The short duration of the study ( 9 months) leads to lack of precise and accurate data as well as improper estimation of treatment outcomes. Further, this study was conducted in a tertiary care hospital, thus only focusing on a small population and lacking generalizability of other in vitro fertilization centres, fertility clinics orhospitals, and hence, lack of involvement of other possible infertile population was another limitation. Letrozole was not used in the current study as it was banned during the period of the study but licensed toward the end of the study as a better choice of ovulation induction drug in infertile PCOS patients.

\section{CONCLUSION}

PCOS is a common endocrine disorder within the age group of 24-27 years, from middle socioeconomic class and mostly residing in urban areas. Most of the infertile PCOS women had obesity concluding it as a major risk factor for this syndrome. Prescription pattern of obese PCOS women mostly involved combination therapy in which most women were given CC pre-treated with OC and CC with metformin pre-administered with OC, while in non-obese PCOS women, most of them were given CC with gonadotropins pre-treated with OC. Lifestyle modifications including diet and exercise are strongly recommended, especially for obese PCOS patients.

\section{ACKNOWLEDGMENT}

The authors would like to thank all the staff including residents, doctors, and nurses of Fertility Department, Bharati Hospital and Research Center, Bharati Vidyapeeth Deemed University, Pune, for helping us in the completion of the study.

\section{CONFLICTS OF INTEREST}

None of the authors have any conflicts of Interest.

\section{REFERENCES}

1. Teede H, Deeks A, Moran L. Polycystic ovary syndrome: A complex condition with psychological, reproductive and metabolic manifestations that impacts on health across the lifespan. BMC Med 2010;8:41

2. Mandrelle K, Kamath MS, Bondu DJ, Chandy A, Aleyamma T, George $\mathrm{K}$, et al. Prevalence of metabolic syndrome in women with polycystic ovary syndrome attending an infertility clinic in a tertiary care hospital in south India. J Hum Reprod Sci 2012;5:26-31.

3. Ramanand SJ, Ghongane BB, Ramanand JB, Patwardhan $\mathrm{MH}$, Ghanghas RR, Jain SS, et al. Clinical characteristics of polycystic ovary syndrome in Indian women. Indian J Endocrinol Metab 2013;17:138-45.

4. Rodin DA, Bano G, Bland JM, Taylor K, Nussey SS. Polycystic ovaries and associated metabolic abnormalities in Indian subcontinent Asian women. Clin Endocrinol (Oxf) 1998;49:91-9.

5. Gambineri A, Pelusi C, Vicennati V, Pagotto U, Pasquali R. Obesity and the polycystic ovary syndrome. Int J Obes Relat Metab Disord 2002;26:883-96.

6. Jyoti N, Jyoti K, Savita RS, Veena SG. Comparision of myo-inositol versus metformin on anthropometric parameters in Polycystic ovarian syndrome in women. Int J Pharm Pharm Sci 2017;9:144-8.

7. Shatha HA, Ali MA, AL-Musawi BJ. Serum irisin and leptin levels 
in obese and non-obese women with Polycystic ovary syndrome with reference to glucose homeostasis. Int J Pharm Pharm Sci 2016;8:276-83.

8. Vrunda D, Parul S, Rajal T, Kruti D. Study of 100 cases of Infertility in Polycystic ovarian syndrome and its management outcome. Int J Med Sci Public Health 2013;2:1041-5.

9. Surekha T, Himabindu Y, Sriharibabu M. Impact of socio-economic status on ovarian reserve markers. J Hum Reprod Sci 2013;6:201-4.

10. Sharon SM, Ricardo A, Teresa S, Calderon-Margalit R, Martha D, Catarina $\mathrm{K}$, et al. Socioeconomic status and Polycystic Ovary Syndrome. J Womens Health 2011;20:413-9.

11. Dasgupta S, Reddy BM. The role of epistasis in the etiology of polycystic ovary syndrome among Indian women: SNP-SNP and SNPenvironment interactions. Ann Hum Genet 2013;77:288-98.

12. Sarkar S, Das M, Mukhopadhyay B, Chakrabarti CS, Majumder PP. High prevalence of metabolic syndrome and its correlates in two tribal populations of India and the impact of urbanization. Indian J Med Res 2006;123:679-86

13. Saxena P, Prakash A, Nigam A, Mishra A. Polycystic ovary syndrome: Is obesity a sine qua non? A clinical, hormonal, and metabolic assessment in relation to body mass index. Indian J Endocrinol Metab 2012;16:996-9.

14. Omokanye L, Ibiwoye-Jaiyeola O, Olatinwo A, Abdul I, Durowade K, Biliaminu S. Polycystic ovarian syndrome: Analysis of management outcomes among infertile women at a public health institution in Nigeria. Niger J Clin Pract 2015;13:44.

15. Orsino A, Van Eyk N, Hamilton J. Clinical features, investigations and management of adolescents with polycystic ovary syndrome. Paediatr Child Health 2005;10:602-8.

16. Kistner RW. Use of clomiphene citrate, HCG and HMG for induction of ovulation in human female. Fertil Steril 1966;17:569-83.

17. Dasari P, Pranahita G. The efficacy of metformin and clomiphene citrate combination compared with clomiphene citrate alone for ovulation induction in infertile patients with PCOS. J Hum Reprod Sci 2009;2:18-22

18. Gill H, Tiwari P, Dabadghao P. Prevalence of polycystic ovary syndrome in young women from north India: A Community-based study. Indian J Endocrinol Metab 2012;16:S389-92. 